\title{
A pilot clinical trial to determine the safety and efficacy of aerosolized hyaluronan as a treatment for COPD
}

Jerome Cantorl

Shuren $\mathrm{Ma}^{2}$

Gerard Turino²

'Department of Pharmaceutical Sciences, St John's University,

${ }^{2}$ Department of Medicine, St Luke's

Mount Sinai Hospital Center,

New York, NY, USA
Correspondence: Jerome Cantor School of Pharmacy and Allied Health Sciences, 8000 Utopia Parkway,

Queens, NY I I439, USA

Tel +l 7189907495

Email jocantor I@gmail.com
This article was published in the following Dove Press journal:

International Journal of COPD

18 September 2017

Number of times this article has been viewed

\begin{abstract}
A novel therapy for COPD involving the use of aerosolized hyaluronan (HA) was tested on a small cohort of COPD patients to determine both its safety and efficacy in reducing levels of desmosine and isodesmosine (DID), biomarkers for elastin degradation. In a 2-week, randomized, double-blind trial, 8 patients receiving $150 \mathrm{kDa} H A$ (mean molecular weight) and 3 others given placebo did not show significant adverse effects with regard to spirometry, electrocardiograms, and hematological indices. Furthermore, measurements of DID in plasma from HA-treated patients indicated a progressive decrease over a 3-week period following initiation of treatment ( $r=-0.98 ; p=0.02$ ), whereas patients receiving placebo showed no reduction in DID ( $r=-0.70 ; p=0.30)$. Measurements of sputum in the HA-treated group also revealed a progressive decrease in DID ( $r=-0.97 ; p=0.03)$, but this finding was limited by the absence of similar measurements in the placebo group. Nevertheless, the results of this small, pilot study support a longer-term trial of HA in a larger population of COPD patients.
\end{abstract}

Keywords: hyaluronan, COPD, desmosine, elastin

\section{Introduction}

The protease-antiprotease concept of lung interstitial injury has encouraged the use of elastase inhibitors as potential treatments for COPD. ${ }^{1,2}$ However, aside from $\alpha-1$ antiprotease (AAP) replacement therapy for AAP-deficient patients, this approach has met with little success, and clinical testing of various inhibitors has failed to produce a successful drug for COPD. ${ }^{3-5}$

As an alternative to this form of treatment, our laboratory is developing a new therapy involving inhalation of hyaluronan (HA), a long-chain polysaccharide. The rationale for using $\mathrm{HA}$ is based on studies indicating that it preferentially binds to lung elastic fibers, prevents elastolysis, and limits airspace enlargement in experimental models of pulmonary emphysema. ${ }^{6-11}$

To further evaluate the therapeutic role of HA, we conducted a 2-week, randomized, double-blind, placebo-controlled, phase 2 a safety trial in 11 patients with COPD. The primary aims of the study were: 1) to determine the safety of administering repeated doses of HA (CTX-100) to subjects with smoking-related COPD and 2) to assess the effects of HA on elastin degradation, as measured by sputum and plasma levels of desmosine and isodesmosine (DID), cross-linking amino acids found only in this protein. The results indicate that inhalation of HA is well tolerated and rapidly reduces lung elastic fiber breakdown, thus supporting a longer-term investigation of the clinical efficacy of this agent. 


\section{Methods}

\section{Study protocol}

Eleven patients were recruited from 2 centers: 9 from Research Associates in Tucson, Arizona and 2 from St Luke'sRoosevelt Hospital Pulmonary Disease Center in New York. The trial (NCT00993707) was conducted under IND number 70299, with Institutional Review Board approval from both organizations, and complied with the standards of Good Clinical Practice. Although the protocol was originally designed as an ascending-dose study (from $0.01 \%$ to $0.03 \%$ HA) involving a second cohort, recruitment was more difficult than anticipated, and the trial was terminated following completion of the initial 14-day treatment regimen.

The patients ranged in age from 40 to 76 and fulfilled the diagnostic criteria of COPD with GOLD grades 2 and 3 with moderate airway obstruction (forced expiratory volume in 1 second above $40 \%$ of predicted) and at least a 10 pack-year history of cigarette smoking (Table 1). Ten were white and 1 was African-American. Informed consent was obtained from all participants.

None were actively smoking at the time of recruitment, and smoking cessation had occurred at least 1 year prior to the study. They were randomly assigned to treatment groups receiving either $0.01 \%$ HA BID ( 8 patients) or matching placebo (3 patients). All patients were instructed to continue their usual bronchodilator therapy during the trial.

Each patient self-administered $3 \mathrm{~mL}$ of aerosolized inhalation solution, using a Pari nebulizer, twice daily for 14 days. The primary safety end points were oxygen saturation, spirometry, lung volumes, physical examination, vital signs, electrocardiogram, and laboratory evaluations (complete blood count, serum chemistries, and urinalysis). Additionally, DID concentrations in sputum and plasma were measured prior to treatment with either CTX-100 or

Table I Patient characteristics

\begin{tabular}{lll}
\hline Characteristic & CTX-100 & Placebo \\
\hline Number of subjects & 8 & 3 \\
Age (years) & $63 \pm 8^{\mathrm{a}}$ & $52 \pm \mathrm{I}^{\mathrm{a}}$ \\
Race & 7 white, I African-American & 3 white \\
$\mathrm{FEV},(\mathrm{L})$ & $1.84 \pm 0.45^{\mathrm{a}}$ & $1.77 \pm 0.44^{\mathrm{a}}$ \\
$\mathrm{DLCO}(\mathrm{mL} / \mathrm{min} / \mathrm{mmHg})$ & $16.6 \pm 8.0^{\mathrm{a}}$ & $19.6 \pm 0.8^{\mathrm{a}}$ \\
Total lung capacity $(\mathrm{L})$ & $7.1 \pm 2.1^{\mathrm{a}}$ & $5.9 \pm 0.5^{\mathrm{a}}$ \\
Smoking Hx & $>10$ pack-years & $>10$ pack-years \\
Active smokers & None & None \\
Inhaled corticosteroids & 4 & None \\
Recent exacerbations & None & None \\
\hline
\end{tabular}

Note: a Mean \pm SD.

Abbreviations: DLCO, carbon monoxide diffusing capacity; FEV , forced expiratory volume in I second; $\mathrm{Hx}$, history; SD, standard deviation. placebo, and again at weekly intervals, including the week following treatment.

\section{HA drug preparation}

The treatment agent (CTX-100) used for the study consisted of $0.01 \%$ HA isolated from Streptococcus equi (150 kDa average molecular weight); dissolved in $3 \mathrm{~mL}$ of isotonic, buffered saline; and packaged in plastic vials under sterile conditions. The placebo consisted of identically packaged isotonic, buffered saline without HA.

\section{Measurement of DID}

Samples were combined with an equal volume of $37 \% \mathrm{HCl}$ and hydrolyzed at $110^{\circ} \mathrm{C}$ for $24 \mathrm{~h}$, then dried under vacuum, suspended in $2 \mathrm{~mL}$ of butanol, acetic acid, and water (4:1:1), applied to a CF1 column, washed, and eluted. Separation of DID was performed with a $2 \times 100 \mathrm{~mm} \mathrm{dC} 18$ column (Waters Corporation, Milford, MA, USA), using a combination of mobile phases: 1) $7 \mathrm{mM}$ heptafluorobutyric acid and $5 \mathrm{mM}$ ammonium acetate in water and 2) $7 \mathrm{mM}$ heptafluorobutyric acid and $5 \mathrm{mM}$ ammonium acetate in $80 \%$ acetonitrile. The elution gradient was programmed linearly from $100 \% \mathrm{~A}$ to 90\% A over a 10-min interval, and the separated crosslinks were analyzed with a TSQ Discovery electrospray tandem mass spectrometer (Thermo Fisher Scientific, Waltham, MA, USA), using selected reaction monitoring of mass-to-charge ratio transitions. The results were quantified by comparison with an external d4-labeled desmosine standard and the sputum results were normalized to total protein, as measured by a Micro BCATM assay kit (Thermo Fisher Scientific, Springfield Township, NJ, USA). ${ }^{12}$

\section{Statistical analysis}

Statistically significant correlations $(p<0.05)$ between DID levels and length of time following treatment were determined by performing a two-tailed $t$-test on the Pearson coefficient $(r)$. Results are expressed as mean \pm standard error of the mean for plasma and sputum DID levels and as mean \pm standard deviation for all other measurements.

\section{Results Safety assessment}

The administration of CTX-100 had no significant effect on spirometry, lung volumes, electrocardiograms, and hematological indices. In particular, forced expiratory volume measurements at 1 second showed no significant changes during the course of the study, which included a 1-week interval posttreatment (Table 2). Similarly, carbon monoxide diffusing capacity remained unchanged during the 2 -week trial 
Table 2 FEV, (liters \pm SD)

\begin{tabular}{llll}
\hline Time point & CTX- $100^{\mathrm{a}}$ & Placebo $^{\mathrm{b}}$ & p-value \\
\hline Pretreatment & $1.84 \pm 0.45$ & $1.77 \pm 0.44$ & 0.92 \\
Week I & $1.80 \pm 0.42$ & $1.75 \pm 0.38$ & 0.92 \\
Week 2 & $1.72 \pm 0.33$ & $1.73 \pm 0.31$ & 1.0 \\
Week 3 & $1.90 \pm 0.51$ & $1.70 \pm 0.29$ & 0.36 \\
\hline
\end{tabular}

Note: ${ }^{\mathrm{a}} \mathrm{N}=8,{ }^{\mathrm{b}} \mathrm{N}=3$.

Abbreviations: $\mathrm{FEV}_{1}$, forced expiratory volume in I second; SD, standard deviation.

(Table 3). Adverse events were generally mild and recurred with greater frequency in the placebo group (Table 4). None could be directly attributed to the inhalation procedure.

\section{Biomarker measurements}

The CTX-100 group showed a progressive decrease in plasma DID levels over a 3-week period following initiation of treatment ( $r=-0.98 ; p=0.02 ;$ Figure 1$)$. In contrast, there was no significant reduction in the placebo group $(r=-0.70 ; p=0.30)$. Sputum DID levels, which specifically reflect lung elastin degradation, also showed a progressive decrease over the same time interval ( $r=-0.97 ; p=0.03$; Figure 2 ). However, this finding was limited by the fact that no patients in the placebo group provided sputum samples for comparison.

\section{Discussion}

The results of this limited safety study indicate that aerosol administration of a $0.01 \%$ solution of HA was well tolerated and did not involve adverse events requiring cessation of treatment. Moreover, there were significant reductions in the levels of DID in plasma and sputum, suggesting that nebulized HA is protecting elastin fibers from degradation and may therefore limit the development of pulmonary emphysema. In comparison to a recent trial involving longterm administration of AAP to COPD patients with AAP deficiency, the reduction in plasma levels of DID in the current study was greater and occurred more rapidly. ${ }^{13}$ The continued drop in both plasma and sputum DID levels during the week following cessation of treatment may reflect the binding of HA to elastic fibers, which persists well beyond its half-life in the lung. ${ }^{8}$

Table 3 DLCO $(\mathrm{mL} / \mathrm{min} / \mathrm{mmHg} \pm \mathrm{SD})$

\begin{tabular}{llll}
\hline Time point & CTX-100 $^{\mathbf{a}}$ & Placebo $^{\mathrm{b}}$ & p-value \\
\hline Pretreatment & $16.6 \pm 8.0$ & $19.6 \pm 0.8$ & 0.54 \\
Week I & $16.2 \pm 7.8$ & $19.8 \pm 1.5$ & 0.46 \\
Week 2 & $15.6 \pm 6.9$ & $19.8 \pm 1.0$ & 0.34 \\
\hline
\end{tabular}

Note: ${ }^{\mathrm{a}} \mathrm{N}=8,{ }^{\mathrm{b}} \mathrm{N}=3$.

Abbreviations: DLCO, carbon monoxide diffusing capacity; SD, standard deviation.
Table 4 Adverse events

\begin{tabular}{ll}
\hline CTX-100 & Placebo \\
\hline Migraine $^{\mathrm{a}}$ & Difficulty breathing $^{\mathrm{a}}$ \\
Joint pain $^{\mathrm{b}}$ & Lung pain $^{\mathrm{a}}$ \\
Chest congestion $^{\mathrm{b}}$ & Profuse sweating $^{\mathrm{a}}$ \\
Fever $^{\mathrm{b}}$ & Coughing $^{\mathrm{b}}$ \\
Intermittent cough $^{\mathrm{b}}$ & Light-headedness $^{\mathrm{b}}$ \\
Pitting edema $^{\mathrm{b}}$ & \\
Diarrhea $^{\mathrm{b}}$ & \\
Light-headedness $^{\mathrm{b}}$ & \\
\hline
\end{tabular}

Note: a Moderate, ${ }^{b}$ mild.

The use of $0.01 \%$ HA was based on US Food and Drug Administration review of the toxicology data submitted by Exhale Therapeutics (Belmont, CA, USA). Focal collections of macrophages were seen in the lungs of rats receiving aerosolized doses equal to or greater than $0.1 \%$. Consequently, the maximum dose allowed for the US Food and Drug Administration-approved clinical trial was 0.03\% BID. This dosage was included in the original trial protocol, and was to be administered to a second cohort, following safe use of the lower dosage. However, constraints put on the study by lack of recruitment of suitable subjects resulted in early termination. Furthermore, certain subjects were unable to spontaneously produce sputum samples (which is not uncommon in COPD patients), thus limiting evaluation of the treatment effect on DID levels in this fluid. Induction of sputum by inhalation of saline was avoided due to potential patient discomfort with this procedure.

The issues encountered in the current study suggest that new strategies need to be implemented in any future trial. With regard to patient recruitment, the number of sites involved in such a study will need to be increased. Preference should be given to sites with large COPD patient populations that are actively recruiting new patients. It may also be possible to access databases compiled by national organizations, such as the $\alpha-1$ Foundation.

Induction of sputum in patients who can tolerate this procedure might also increase the number of samples available for measurement of DID. The advantage of measuring DID levels in sputum is predicated on the specificity of this parameter for pulmonary elastic fiber degradation. Whereas plasma and urine contain DID from the multiple sites other than the lung, particularly the cardiovascular system, sputum is derived entirely from the lung. While it may be argued that sputum is also representative of elastic fiber injury in the upper airways, reductions in DID levels anywhere in the lung would nevertheless be indicative of the efficacy of HA. The more important issue is whether the aerosolized HA is 


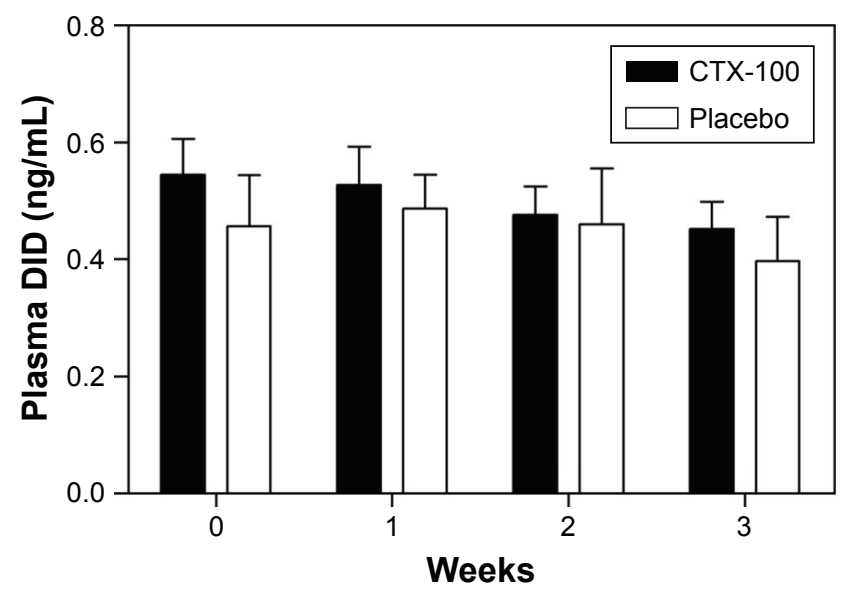

\begin{tabular}{|l|l|l|l|l|l|l|l|}
\hline Group & N & Pre & Week 1 & Week 2 & Week 3 & Pearson's $\boldsymbol{p}$ & $\boldsymbol{p}$-value \\
\hline CTX-100 & 8 & $0.55 \pm 0.06$ & $0.53 \pm 0.07$ & $0.48 \pm 0.05$ & $0.45 \pm 0.05$ & -0.98 & 0.02 \\
\hline Placebo & 3 & $0.46 \pm 0.09$ & $0.49 \pm 0.06$ & $0.46 \pm 0.10$ & $0.40 \pm 0.08$ & -0.70 & 0.30 \\
\hline
\end{tabular}

Figure I Graph showing progressive reduction in plasma DID levels following treatment with CTX-I00 $(r=-0.98 ; p=0.02)$.

Note: Data are expressed as mean \pm SEM.

Abbreviations: DID, desmosine and isodesmosine; SEM, standard error of the mean.

being delivered to the distal lung as well as the upper airways. In response to this question, our laboratory has shown that aerosolized fluorescein-labeled HA (150 kDa molecular weight) preferentially binds to alveolar wall elastic fibers,

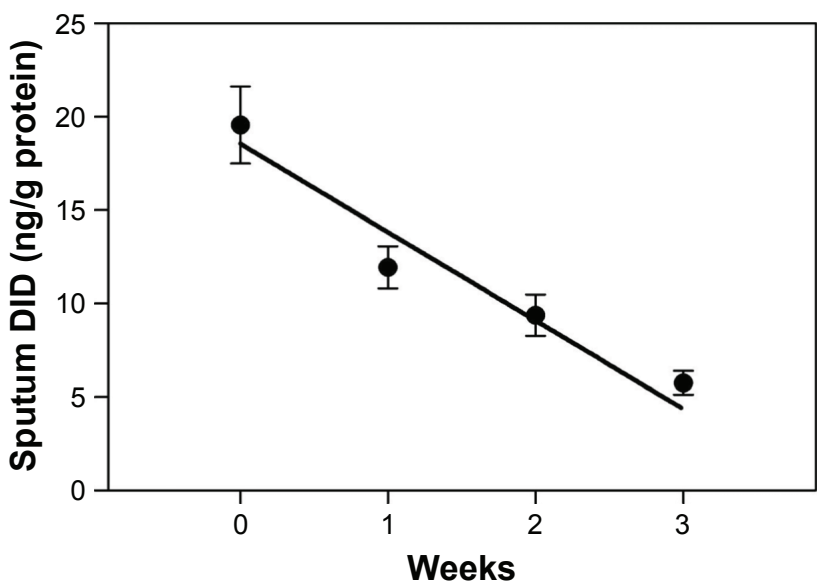

\begin{tabular}{|l|l|l|}
\hline Time point & $\mathbf{C T X}-\mathbf{1 0 0}(\mathbf{N})$ & Placebo (N) \\
\hline Pretreatment & $19.6 \pm 2.1(7)$ & $12.0 \pm 6.3(2)$ \\
\hline Week 1 & $12.0 \pm 1.1(6)$ & - \\
\hline Week 2 & $9.4 \pm 1.1(5)$ & - \\
\hline Week 3 & $5.8 \pm 0.6(2)$ & - \\
\hline
\end{tabular}

Figure 2 Graph showing significant negative correlation between sputum DID levels and time following treatment with CTX-100 ( $r=-0.97 ; p=0.03)$.

Note: Data are expressed as mean \pm SEM.

Abbreviations: DID, desmosine and isodesmosine; SEM, standard error of the mean. presumably preventing interaction with injurious agents such as elastases and oxidants. ${ }^{8}$

With regard to the commercialization of this form of treatment, aerosolized preparations of HA compounds have been used as therapeutic aerosols for upper respiratory disorders in countries outside the United States for several years, but never tested for efficacy in COPD. In Italy, the compound YABRO $^{\text {TM }}$ (IBSA Corporation, Lugano, Switzerland) has been used to treat respiratory ailments for at least 5 years with more than 300,000 prescriptions already issued. HA has also been used in patients with cystic fibrosis as an additive to hypertonic saline therapy. These compounds are prepared in higher molecular weight forms (300-1,000 kDa), but our data (unpublished) suggest that the reduced viscosity of $150 \mathrm{kDa}$ HA lowers the diameter of the aerosol particles, thereby facilitating their access to elastic fibers in the distal lung, whose destruction is a central feature of pulmonary emphysema.

While there are a number of animal studies indicating proinflammatory events associated with low-molecular-weight HA, the findings are generally associated with preexisting acute lung injury, and so may not be representative of the chronic, subacute inflammatory process associated with COPD. ${ }^{14-16}$ Moreover, the current clinical trial showed no evidence of HA-induced inflammation, and long-term treatment of smoke-exposed animals with aerosolized HA did not show 
increased numbers of leukocytes, in either bronchoalveolar lavage fluid or histological sections of the lung. ${ }^{10}$

The concept of using HA to treat pulmonary emphysema is based on a series of experiments designed to determine whether agents other than elastases were capable of inducing pulmonary emphysema. In one such study, pretreatment of the lung with hyaluronidase significantly increased elastaseinduced airspace enlargement. ${ }^{9}$ Experiments were then undertaken to examine the effect of HA itself on this model of emphysema. Animals treated with aerosolized HA prior to instillation of elastase showed significantly less airspace enlargement than controls treated with elastase alone. ${ }^{6-9}$ Similar results were obtained with a model of cigarette smoke-induced emphysema, even when HA treatment was delayed for 1 month following initiation of smoke exposure, suggesting that active smokers might also benefit from this treatment. ${ }^{10,11}$

The use of aerosolized fluorescein-labeled HA showed that this polysaccharide preferentially binds to elastic fibers, suggesting that it acts as a physical barrier to cells and enzymes responsible for elastolysis. ${ }^{6,8}$ Such protection should occur naturally since endogenous $\mathrm{HA}$ is embedded in elastic fibers. ${ }^{17}$ Moreover, a recent study suggests that the proximity of HA to elastic fibers may improve the mechanical properties of the lung by reducing the uneven distribution of forces in the extracellular matrix. ${ }^{18}$ These findings may explain the observed correlation between reduced lung levels of $\mathrm{HA}$ and increased severity of disease in AAP deficiency-induced COPD. ${ }^{19}$

Since elastic fiber breakdown may be a final common pathway in COPD, HA might be effective against a variety of injurious agents involved in the pathogenesis of this disease. In contrast to other proposed treatments, such as specific elastase inhibitors, HA might provide broader protection of the lung with fewer potential side effects. Furthermore, the generally slow progression of alveolar wall damage in COPD suggests that even small decreases in elastic fiber injury could significantly reduce the risk of respiratory failure.

\section{Disclosure}

Both Jerome Cantor and Gerard Turino have a financial interest in MatRx Therapeutics, a company that is developing HA for use in COPD. The authors report no other conflicts of interest in this work.

\section{References}

1. Barnes PJ, Shapiro SD, Pauwels RA. Chronic obstructive pulmonary disease: molecular and cellular mechanisms. Eur Respir J. 2003;22: 672-678.

2. Shapiro SD. Proteinases in chronic obstructive pulmonary disease. Biochem Soc Trans. 2002;30:98-102.

3. Kuna P, Jenkins M, O’Brien CD, Fahy WA. AZD9668, a neutrophil elastase inhibitor, plus ongoing budesonide/formoterol in patients with COPD. Respir Med. 2012;106:531-539.

4. Lucas SD, Costa E, Guedes RC, Moreira R. Targeting COPD: advances on low-molecular-weight inhibitors of human neutrophil elastase. Med Res Rev. 2013;33(Suppl 1):E73-E101.

5. Groutas WC, Dou D, Alliston KR. Neutrophil elastase inhibitors. Expert OpinTher Pat. 2011;21:339-354.

6. Cantor JO, Shteyngart B, Cerreta JM, et al. The effect of hyaluronan on elastic fiber injury in vitro and elastase-induced airspace enlargement in vivo. Proc Soc Exp Biol Med. 2000;225:65-71.

7. Cantor JO, Cerreta JM, Armand G, Turino GM. Aerosolized hyaluronic acid decreases alveolar injury induced by human neutrophil elastase. Proc Soc Exp Biol Med. 1998;217:471-475.

8. Cantor JO, Cerreta JM, Armand G, Turino GM. Further investigation of the use of intratracheally administered hyaluronic acid to ameliorate elastase-induced emphysema. Exp Lung Res. 1997;23: 229-244.

9. Cantor JO, Cerreta JM, Keller S, Turino GM. Modulation of airspace enlargement in elastase-induced emphysema by intratracheal instillment of hyaluronidase and hyaluronic acid. Exp Lung Res. 1995;21: 423-436.

10. Cantor JO, Cerreta JM, Ochoa M, et al. Aerosolized hyaluronan limits airspace enlargement in a mouse model of cigarette smoke-induced pulmonary emphysema. Exp Lung Res. 2005;31:417-430.

11. Cantor JO, Cerreta J, Ochoa M, et al. Therapeutic effects of hyaluronan on smoke-induced elastic fiber injury: does delayed treatment affect efficacy? Lung. 2011;189:51-56.

12. Ma S, Turino GM, Hayashi T, et al. Stable deuterium internal standard for the isotope-dilution LC-MS/MS analysis of elastin degradation. Anal Biochem. 2013;440:158-165.

13. Ma S, Lin YY, Cantor JO, et al. The effect of alpha-1 protease inhibitor (A1PI) on biomarkers of elastin degradation in alpha-1 antitrypsin deficiency (AATD): an analysis of the RAPID/RAPID Extension trials. Chronic Obstr Pulm Dis. 2017;4:34-44.

14. Garantziotis S, Li Z, Potts EN, et al. Hyaluronan mediates ozoneinduced airway hyperresponsiveness in mice. J Biol Chem. 2009;284: 11309-11317.

15. Nadkarni PP, Kulkarni GS, Cerreta JM, et al. Dichotomous effect of aerosolized hyaluronan in a hamster model of endotoxin-induced lung injury. Expert Lung Res. 2005;31:807-818.

16. Noble PW, Jiang D. Matrix regulation of lung injury, inflammation, and repair: the role of innate immunity. Proc Am Thorac Soc. 2006;3: 401-404.

17. Baccarani-Contri M, Vincenzi D, Cicchetti F, et al. Immunocytochemical localization of proteoglycans within normal elastin fibers. Eur J Cell Biol. 1990;53:305-312.

18. Takahashi A, Majumdar A, Parameswaran H, et al. Proteoglycans maintain lung stability in an elastase-treated mouse model of emphysema. Am J Respir Cell Mol Biol. 2014;51:26-33.

19. Cantor J, Armand G, Turino G. Lung hyaluronan levels are decreased in alpha-1antiprotease deficiency COPD. Respir Med. 2015;109: 656-659. 


\section{Publish your work in this journal}

The International Journal of COPD is an international, peer-reviewed journal of therapeutics and pharmacology focusing on concise rapid reporting of clinical studies and reviews in COPD. Special focus is given to the pathophysiological processes underlying the disease, intervention programs, patient focused education, and self management protocols.

This journal is indexed on PubMed Central, MedLine and CAS. The manuscript management system is completely online and includes a very quick and fair peer-review system, which is all easy to use. Visit http://www.dovepress.com/testimonials.php to read real quotes from published authors.

Submit your manuscript here: http://www.dovepress.com/international-journal-of-chronic-obstructive-pulmonary-disease-journal 